\title{
Polarimetric measures of selected variable stars ${ }^{\star}$
}

\author{
N. M. Elias $\mathrm{II}^{1,2}$, R. H. Koch ${ }^{3, \star \star}$, and R. J. Pfeiffer ${ }^{4}$ \\ 1 Zentrum für Astronomie der Universität Heidelberg, Landessternwarte, Königstuhl 12, 69117 Heidelberg, Germany \\ e-mail: n.elias@lsw.uni-heidelberg.de \\ 2 Max-Planck-Institut für Astronomie, Königstuhl 17, 69117 Heidelberg, Germany \\ 3210 Roberts Road, Ardmore, PA 19003, USA \\ e-mail: rhkoch@earthlink.net \\ 4 Department of Physics, The College of New Jersey, Ewing, NJ 08628-0718, USA \\ e-mail: pfeiffer@tcnj .edu
}

Received 6 February 2008 / Accepted 1 July 2008

\section{ABSTRACT}

\begin{abstract}
Aims. The purpose of this paper is to summarize and interpret unpublished optical polarimetry for numerous program stars that were observed over the past decades at the Flower and Cook Observatory (FCO), University of Pennsylvania. We also make the individual calibrated measures available for long-term comparisons with new data.

Methods. We employ three techniques to search for intrinsic variability within each dataset. First, when the observations for a given star and filter are numerous enough and when a period has been determined previously via photometry or spectroscopy, the polarimetric measures are plotted versus phase. If a statistically significant pattern appears, we attribute it to intrinsic variability. Second, we compare means of the FCO data to means from other workers. If they are statistically different, we conclude that the object exhibits long-term intrinsic variability. Third, we calculate the standard deviation for each program star and filter and compare it to the standard deviation estimated from comparable polarimetric standards. If the standard deviation of the program star is at least three times the value estimated from the polarimetric standards, the former is considered intrinsically variable. All of these statements are strengthened when variability appears in multiple filters.

Results. We confirm the existence of an electron-scattering cloud at $L_{1}$ in the $\beta$ Per system, and find that LY Aur and HR 8281 possess scattering envelopes. Intrinsic polarization was detected for Nova Cas 1993 as early as day +3 . We detected polarization variability near the primary eclipse of $32 \mathrm{Cyg}$. There is marginal evidence for polarization variability of the $\beta$ Cepheid type star $\gamma$ Peg. The other objects of this class exhibited no variability. All but one of the $\beta$ Cepheid objects (ES Vul) fall on a tight linear relationship between linear polarization and $E(B-V)$, in spite of the fact that the stars lay along different lines of sight. This dependence falls slightly below the classical upper limit of Serkowski, Mathewson, and Ford.
\end{abstract}

Key words. polarization - binaries: close - Cepheids - circumstellar matter - accretion, accretion disks - dust, extinction

\section{Introduction}

The polarimetric program at the Flower and Cook Observatory (FCO) was terminated in 1996 after 27 years. Two recent papers (Koch \& Clarke 2005; Koch 2006) summarized the observing, reduction, and calibration procedures. They presented almost 1500 linear and circular polarization standard-star observations. We use those quantities in this work to infer the variability/nonvariability of FCO program targets. Results for a number of these objects have been presented by an assortment of authors, but a significant amount of polarization data has yet to be published.

The program stars described here are close binaries (spectroscopic, eclipsing, or ellipsoidal) suspected of having scattering envelopes, and $\beta$ Cepheid non-radial pulsators. We characterize the polarization measures by their means, standard deviations, and reduced standard deviations. All of the measures have been

\footnotetext{
$\star$ The table, which contains the polarization observations of the program stars discussed in this paper, is only available in electronic form at the CDS via anonymous ftp to

cdsarc.u-strasbg.fr $(130.79 .128 .5)$ or via

http://cdsweb.u-strasbg.fr/cgi-bin/qcat?J/A+A/489/911

$\star \star$ Professor Emeritus, Department of Physics and Astronomy, University of Pennsylvania, 209 S. 33rd Street, Philadelphia, PA 191046394, USA.
}

corrected for instrumental polarization as explained in Koch \& Clarke (2005) and Koch (2006). The reduced standard deviation (or, standard deviation of the mean) is the standard deviation (the error estimate of a single datum) divided by the square root of the number of measurements. We interpret the present and archival polarization measures for each object, and also compare them to their interstellar extinction measures. The individual observations will be useful to future workers, so we make them available in an on-line table ${ }^{\star}$.

\section{Algorithms to determine polarization variability}

Because the program stars reported here are light and velocity variables, it is natural to wonder if they are also polarization variables. For several of them, phase-locked polarization variability is evident, demonstrating intrinsic polarization. Periodograms (e.g., Scargle 1982) can be used to search for other periods, if there is a sufficient number of data. Comparing historical measures to the ones reported here often shows significant differences (based on reduced standard deviations), which means that long-term intrinsic variability is a reasonable inference even in the absence of a phase-locked pattern. For a number of the objects, however, poor phase coverage and observing errors prevent either of these simple techniques from providing a complete 


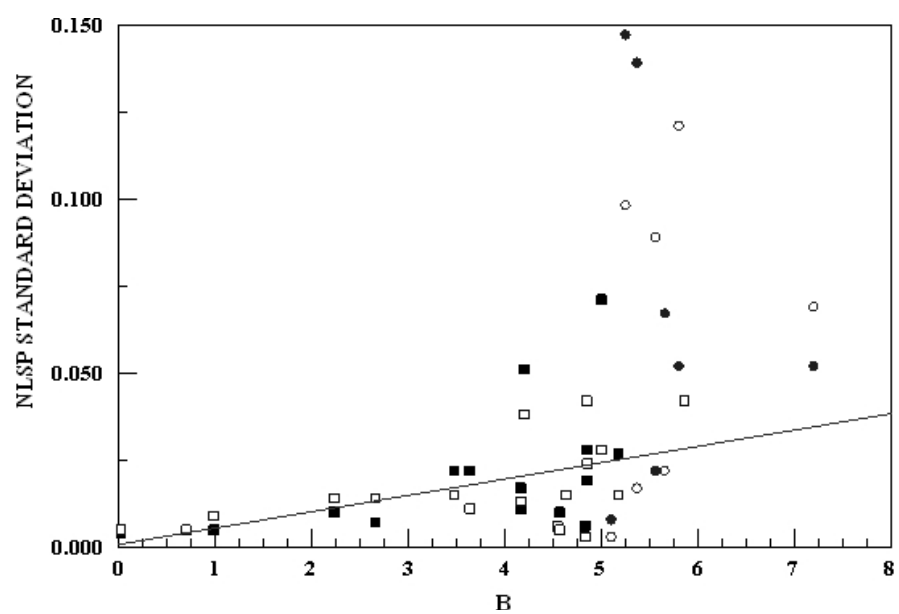

Fig. 1. The calculated standard deviations of nominal NLSP observations (\%) using a Johnson-Morgan $B$ filter. The squares represent null standards, and the circles represent non-null standards. The filled and open symbols refer to NLSP $q$ and NLSP $u$, respectively. The straight line represents Eq. (1) in the text.

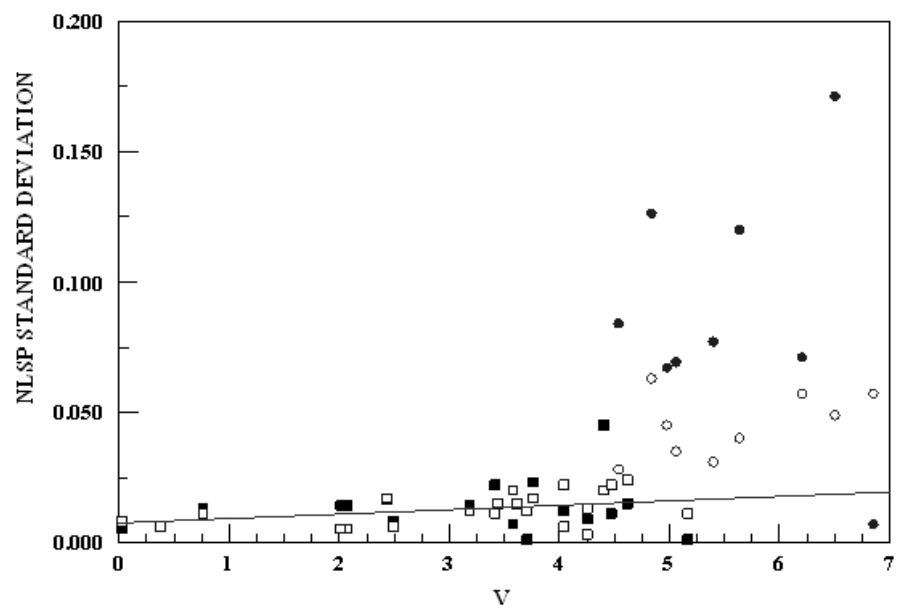

Fig. 2. The calculated standard deviations of nominal NLSP observations (\%) using a Johnson-Morgan $V$ filter. The symbols are the same as in Fig. 1. The straight line represents Eq. (2) in the text.

picture. A third criterion for examining possible intrinsic variability is described below.

If standard deviations have been calculated for a large ensemble of null and non-null polarization standards, it should be possible to compare those results to the program-star standard deviations of similar brightness. We prefer not to make weak statements of variability, even if it means that we miss real variability. Therefore, if the standard deviation of a program star is less than a factor of three greater than the standard deviation estimated from polarization standards, it will not be declared variable. Regardless of the results of this test, we also considered the criteria developed by Stewart (1991).

In Figs. 1-3, we show the standard deviations of the normalized linear Stokes parameters (NLSP; $q$ and $u$ ) for the standard stars versus apparent magnitude. Sometimes, the linear polarization $p=\sqrt{q^{2}+u^{2}}$ and the position angle $\theta=\frac{1}{2} \tan ^{-1} u / q$ will be mentioned. As expected for an observing procedure that hardly ever varied, these values depend significantly upon magnitude. The magnitudes are somewhat ill-defined since no corrections were applied for atmospheric extinction, and targets were not observed at unique airmasses. From long-term photometric

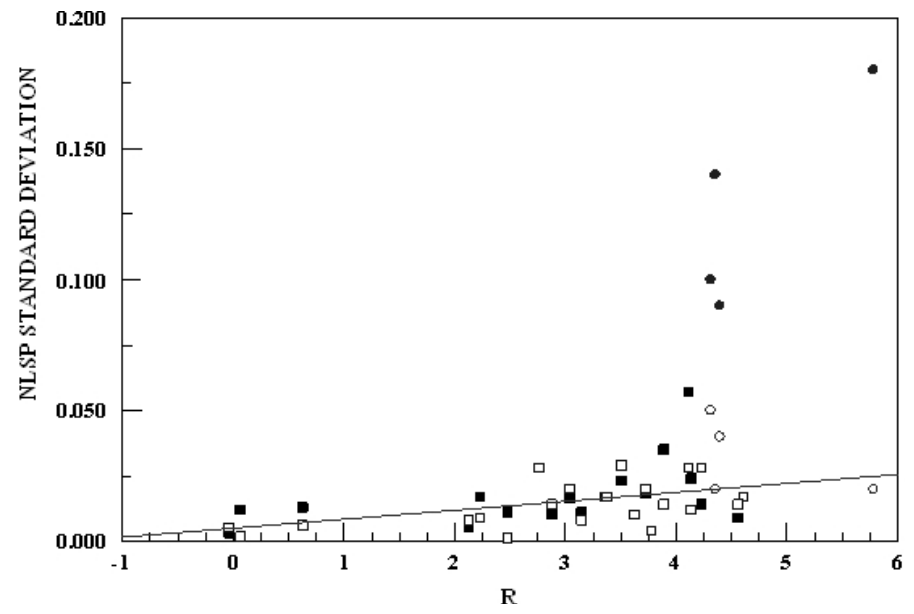

Fig. 3. The calculated standard deviations of nominal NLSP observations (\%) using a Johnson-Morgan $R$ filter. The symbols are the same as in Fig. 1. The straight line represents Eq. (3) in the text.

programs, it is known that typical extinction coefficients are $\approx 0.53(B), 0.37(V)$, and $0.22(R)$. Variations from these values were sometimes large. Except for $\delta$ Ori, no polarization measures were obtained at airmasses greater than two. Also, no extinction-dependent variations of polarization were observed for $\delta$ Ori itself.

Perfoming weighted least-squares fits on the NLSP standard deviations versus magnitude of the null standards, we obtain the following linear relationships:

$\sigma_{B}=0.0009 \%( \pm 0.0055 \%)+0.0047 \%( \pm 0.0014 \%) B$
$\sigma_{V}=0.0076 \%( \pm 0.0029 \%)+0.0017 \%( \pm 0.0008 \%) V$

and

$\sigma_{R}=0.0052 \%( \pm 0.0036 \%)+0.0034 \%( \pm 0.0011 \%) R$.

These equations are indicated in Figs. $1-3$. The total number of $B, V$, and $R$ measures are 322,240 , and 236 , respectively. The parenthesized numbers are the $1 \sigma$ errors of the fit parameters. We found that the dependences were statistically identical for the NLSP $q$ and NLSP $u$ values. It would have been possible to form a mean relation valid for all bandpasses, but we did not do so because the polarization of a few binaries is very different among all three filters.

When the relatively few and typically fainter non-null standard stars are included along with the null standards, the $B$ and $V$ formulae become

$\sigma_{B}=-0.0099 \%( \pm 0.0116 \%)+0.0096 \%( \pm 0.0015 \%) B$

and

$\sigma_{V}=-0.0130 \%( \pm 0.0092 \%)+0.0107 \%( \pm 0.0022 \%) V$

Only four non-null standard stars were measured with the $R$ filter. Three are light variables and two are polarization variables (Bastien et al. 2007), so we did not perform another fit that included them. The total number of $B$ and $V$ measures is 398 and 292, respectively. We find steeper slopes and slightly increased coefficient errors.

In general, the non-null standards are fainter than the null ones, but counting times were not increased to take account of this difference. The observing practice for the final 15 years of the program invested $900 \mathrm{~s}$ and $300 \mathrm{~s}$ in the star+sky and sky 


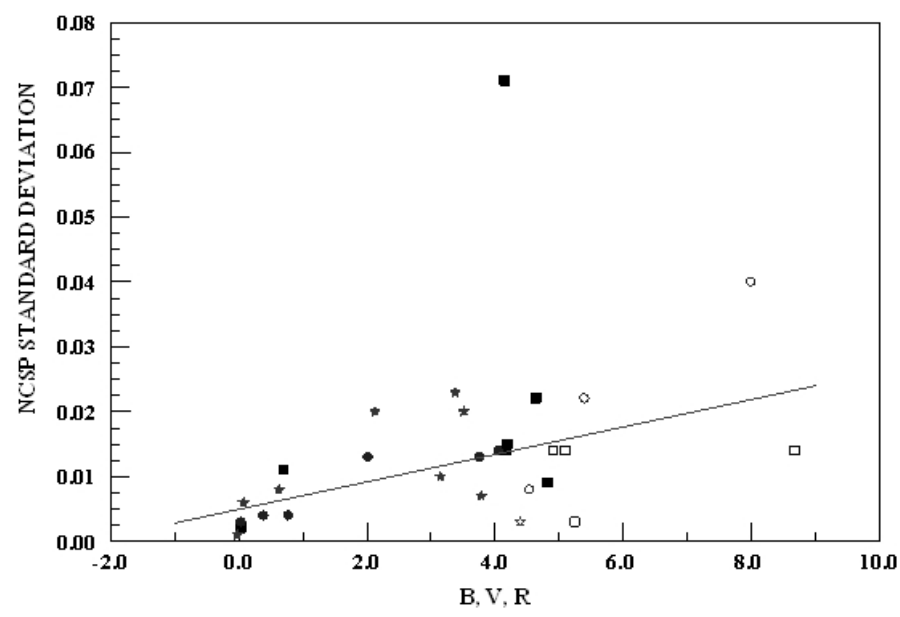

Fig. 4. The calculated standard deviations of nominal NCSP observations (\%) using a Johnson-Morgan $B$ (squares), $V$ (circles), or $R$ (stars) filter. The filled symbols refer to null standards, and the open ones refer to non-null standards. The straight line represents Eq. (6) in the text.

data, respectively, irrespective of target brightness. Since observing practice limited azimuthal sampling of the focused beam to nine or ten settings, the polarization waveform for a non-null standard was always under-sampled and, of course, the measure at each azimuth is afflicted with observing error. The impact of shot noise dominating measures for faint stars is also evident in the figures.

For a highly-polarized source, the errors of the NLSPs will be larger than for a feebly polarized target because of shot noise and azimuthal undersampling, and it might be expected that the calibrating equations lack a necessary dependence on measured polarization value. This possibility was examined but the evidence is confused for two reasons: the polarized standards are not so numerous as the null standards, and some of the strongest polarized standards have unaccountably small standard deviations. Even though Eqs. (1)-(3) may underestimate the errors of fainter stars compared Eqs. (4) and (5), we employ the former because 1) they exhibit tighter linear relationships; and 2) the same stars were found to be variable using both types of fits.

The normalized circular Stokes parameters (NCSPs; $v$ ) for standard stars were examined in the same way as the more numerous NLSPs. In Fig. 4, we present the standard deviations versus magnitude of all null and non-null standards for all filters as a single dataset. The best representation for all the NCSP standard deviations is

$\sigma_{M}=0.0050 \%( \pm 0.0023 \%)+0.0021 \%( \pm 0.0006 \%) M$,

where $M=B, V$, or $R$. The total number of measures is 893 . As for NLSP, a target is deemed an NCSP variable if its standard deviation is more than three times larger than the standard deviation of a standard star with a similar brightness. No NCSP value is as large as any non-null standard NLSP.

\section{Close binaries}

Close binaries formed the core of an FCO observing program primarily because of interest in scattering from circumstellar shells enveloping one or both of the stars. We present Table 1, which gives weighted means, reduced standard deviations, and numbers of NLSP measures. The reduced standard deviations must be multiplied by the square root of the number of measurements for comparison with the standard deviations of polarized standard stars. If an object was observed only once with a given filter, there is no measure of precision. The stars are presented in order of increasing right ascension. Brief remarks for most of the targets appear in the following subsections. In order to implement the error calibrations, we employed the atlas of Kreiner et al. (2001), which gives modern ephemerides, magnitudes at maximum light, and eclipse depths. For every object, the interstellar polarization was calculated by interpolation in galactic latitude, galactic longitude, and distance for field stars within a radius of $5^{\circ}$.

\subsection{AP PsC}

The NLSP standard deviations fit those of the non-null standards quite well so the object would appear to be constant. The observations, however, can be fitted to a double sinusoid in the 96-day orbital period with the most negative values ocurring half a cycle apart. The average deviation from the sinusoid is $\approx 0.02 \%$. Perhaps the distorted K2 III component contributes to the signal. Most of the polarization is interstellar. The evidence for a variable NCSP is marginal at best.

\section{2. $\zeta$ And}

The pair of $V$ measures is statistically identical. The large formal standard deviation of NLSP $u$ in the $R$ filter is due to the large dispersion among a few measures that was caused by insufficient integration times.

\section{3. $\beta \operatorname{Per}($ Algol)}

The FCO observations are from the 1987-1988 through 1990-1991 seasons. They represent a trivial case for demonstrating intrinsic NLSP variability by inspection. Although phase coverage is not uniform, each parameter actually tracks the very low-amplitude, phase-locked modulations already described by Kemp et al. (1981) from earlier seasons (see Fig. 5). The modulation of NLSP $q$ is a factor of three smaller than of NLSP $u$ and is not displayed.

The 1976-1980 Oregon observations by Kemp et al. were made with a $B$ filter. The FCO NLSP amplitude is larger than the Oregon one and each dataset drops to very nearly $0.00 \%$ at the stellar conjunctions. The Oregon authors presented two possible scenarios to account for their observations. The first one relies on a Coriolis-distorted photosphere of the cool star filling its Roche Lobe, leading to an asymmetry of NLSP at the stellar elongations. A more likely explanation produces NLSP by reflection from a concentration of scatterers near the $L_{1}$ point.

In a limited way, it is possible to determine if the amplitude change between Oregon and FCO measures is intrinsic to the object or due only to the bandpass difference. We start with a series of assumptions: 1) the scatterers are electrons; 2) the contribution from the third star of the Algol system may be neglected because its inverse-square-diluted light is meager compared to that of the eclipsing pair; and 3) the light ratios for the eclipsing stars are those given by Kim (1980).

Because the polarization is essentially $0.00 \%$ at both stellar conjunctions for both datasets, it is sufficient to look at the inverse-square-diluted light scattered from the $L_{1}$ point for phases 0.25 and 0.75 . One can calculate the combined light ratio $l_{B} / l_{V}$ of both stars incident on the scattering region, including the radiation "reflected" from the interior photosphere of the cool star after being absorbed from the hot star, and compare it to the 
Table 1. The weighted means of the NLSPs and NCSPs, together with the reduced standard deviations and numbers of observations. The reduced standard deviations must be multipled by the square root of the number of measurements to compare them with the standard-deviation equations.

\begin{tabular}{|c|c|c|c|c|c|c|c|c|c|}
\hline Name & Filter & $\begin{array}{c}q \\
(\%)\end{array}$ & $\begin{array}{c}\sigma_{q} \\
(\%)\end{array}$ & $\begin{array}{c}u \\
(\%)\end{array}$ & $\begin{array}{c}\sigma_{u} \\
(\%)\end{array}$ & $\overline{N_{\text {lin }}}$ & $\begin{array}{c}v \\
(\%)\end{array}$ & $\begin{array}{c}\sigma_{v} \\
(\%)\end{array}$ & 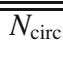 \\
\hline BC Psc / HD 28 & $V$ & -0.03 & - & +0.01 & - & 1 & - & - & 0 \\
\hline AP Psc / HD 352 & $V$ & -0.23 & 0.07 & -0.30 & 0.04 & 5 & +0.09 & 0.08 & 5 \\
\hline \multirow[t]{4}{*}{$\zeta$ And / HD 4502} & $U$ & -0.01 & - & -0.07 & - & 1 & - & - & 0 \\
\hline & $B$ & +0.02 & - & +0.02 & - & 1 & - & - & 0 \\
\hline & $V$ & -0.02 & 0.02 & +0.05 & 0.03 & 2 & - & - & 0 \\
\hline & $R$ & +0.01 & 0.02 & -0.10 & 0.09 & 3 & - & - & 0 \\
\hline$\beta$ Per / HD 19356 & $V$ & -0.0021 & 0.0009 & -0.007 & 0.001 & 53 & +0.0006 & 0.0009 & 47 \\
\hline SZ Cam / HD 25638 & $B$ & +1.30 & 0.04 & -5.84 & 0.06 & 8 & - & - & 0 \\
\hline \multirow[t]{4}{*}{ KS Per / HD 30353} & $U$ & +1.23 & - & -1.77 & - & 1 & - & - & 0 \\
\hline & $B$ & +0.89 & 0.09 & -1.59 & 0.05 & 3 & - & - & 0 \\
\hline & $V$ & +0.90 & 0.08 & -1.63 & 0.04 & 6 & - & - & 0 \\
\hline & $R$ & +0.73 & - & -1.52 & - & 1 & - & - & 0 \\
\hline \multirow[t]{4}{*}{$\epsilon$ Aur / HD 31964} & $U$ & +0.33 & - & -1.95 & - & 1 & - & - & 0 \\
\hline & $B$ & +0.79 & 0.07 & -2.03 & 0.01 & 2 & - & - & 0 \\
\hline & $V$ & +0.81 & - & -1.98 & - & 1 & - & - & 0 \\
\hline & $R$ & +0.78 & - & -1.87 & - & 1 & - & - & 0 \\
\hline$\alpha$ Aur / HD 34029 & $B$ & -0.03 & 0.04 & -0.04 & 0.01 & 5 & - & - & 0 \\
\hline AR Aur / HD 34364 & "white" & -0.007 & 0.002 & -0.010 & 0.002 & 57 & -0.01 & 0.01 & 13 \\
\hline$\eta$ Ori / HD 35411 & B & +0.060 & 0.005 & +0.037 & 0.005 & 17 & - & - & 0 \\
\hline \multirow[t]{4}{*}{ LY Aur / HD 35921} & $U$ & +0.46 & 0.09 & -1.55 & 0.12 & 5 & - & - & 0 \\
\hline & $B$ & +0.49 & 0.02 & -1.68 & 0.03 & 16 & - & - & 0 \\
\hline & $V$ & +0.50 & 0.02 & -1.75 & 0.02 & 37 & - & - & 0 \\
\hline & $R$ & +0.37 & 0.10 & -1.59 & 0.08 & 5 & - & - & 0 \\
\hline$\delta$ Ori / HD 36485 & B & -0.160 & 0.006 & +0.029 & 0.004 & 69 & - & - & 0 \\
\hline \multirow{4}{*}{ V395 Aur / HD 43246} & $U$ & +0.28 & 0.02 & +0.18 & 0.02 & 3 & +0.5 & 0.3 & 3 \\
\hline & $B$ & +0.30 & 0.01 & +0.15 & 0.01 & 3 & +0.2 & 0.1 & 3 \\
\hline & $V$ & +0.23 & 0.05 & +0.12 & 0.02 & 3 & +0.07 & 0.01 & 3 \\
\hline & $R$ & +0.25 & 0.06 & +0.16 & 0.02 & 3 & +0.15 & 0.01 & 3 \\
\hline \multirow[t]{3}{*}{ UX Mon / HD 65607} & $U$ & +1.67 & - & -0.95 & - & 1 & - & - & 0 \\
\hline & $B$ & +0.34 & - & -0.42 & - & 1 & - & - & 0 \\
\hline & $V$ & +0.34 & 0.06 & -0.16 & 0.01 & 3 & - & - & 0 \\
\hline \multirow[t]{4}{*}{ TX UMa / HD 93033} & $U$ & +0.01 & 0.02 & -0.00 & 0.02 & 3 & - & - & 0 \\
\hline & $B$ & -0.01 & 0.01 & -0.03 & 0.03 & 3 & - & - & 0 \\
\hline & $V$ & +0.01 & 0.02 & -0.03 & 0.02 & 3 & - & - & 0 \\
\hline & $R$ & -0.03 & 0.01 & -0.03 & 0.01 & 2 & - & - & 0 \\
\hline \multirow[t]{2}{*}{$\delta \mathrm{Lib} / \mathrm{HD} 132742$} & $B$ & -0.21 & 0.01 & +0.04 & 0.02 & 6 & - & - & 0 \\
\hline & $V$ & -0.26 & - & +0.09 & - & 1 & - & - & 0 \\
\hline$\alpha \mathrm{CrB} / \mathrm{HD} 139006$ & $B$ & +0.11 & 0.03 & -0.09 & 0.04 & 7 & - & - & 0 \\
\hline V1143 Cyg / HD 185912 & $B$ & +0.15 & 0.05 & -0.08 & 0.07 & 6 & - & - & 0 \\
\hline V380 Cyg / HD 187879 & $B$ & +0.14 & 0.02 & -0.10 & 0.04 & 5 & - & - & 0 \\
\hline 31 Cyg / HD 192577 & $B$ & +0.18 & 0.04 & -0.01 & 0.04 & 2 & - & - & 0 \\
\hline \multirow[t]{4}{*}{32 Cyg / HD 192909} & $U$ & +0.16 & 0.07 & -0.20 & 0.07 & 9 & - & - & 0 \\
\hline & $B$ & +0.06 & 0.03 & -0.15 & 0.03 & 17 & - & - & 0 \\
\hline & $V$ & +0.09 & 0.02 & -0.03 & 0.02 & 11 & - & - & 0 \\
\hline & $R$ & +0.04 & 0.03 & -0.01 & 0.03 & 10 & - & - & 0 \\
\hline HR 8281 / HD 206267 & $B$ & +0.55 & 0.06 & +1.15 & 0.03 & 7 & - & - & 0 \\
\hline$\delta$ Cap / HD 207098 & $B$ & -0.21 & - & -0.04 & - & 1 & - & - & 0 \\
\hline \multirow[t]{4}{*}{ AH Cep / HD 216014} & $B 1$ & -0.73 & 0.02 & +1.46 & 0.02 & 20 & - & - & 0 \\
\hline & $B 2$ & -0.81 & 0.01 & +1.68 & 0.01 & 36 & - & - & 0 \\
\hline & $V$ & -0.64 & - & +1.49 & - & 1 & - & - & 0 \\
\hline & $R$ & -0.56 & - & +1.28 & - & 1 & - & - & 0 \\
\hline CW Cep / HD 218066 & $B$ & +0.08 & 0.02 & +0.45 & 0.02 & 22 & +0.00 & 0.02 & 14 \\
\hline AR Cas / HD 221253 & $B$ & +0.03 & 0.03 & -0.06 & 0.02 & 7 & - & - & 0 \\
\hline$\omega$ Psc / HD 224617 & $B$ & -0.05 & 0.09 & -0.04 & 0.02 & 6 & - & - & 0 \\
\hline \multirow[t]{4}{*}{ V705 Cas } & $U$ & -0.91 & - & +1.25 & - & 1 & +3.40 & - & 1 \\
\hline & $B$ & -1.16 & - & +1.78 & - & 1 & +3.40 & - & 1 \\
\hline & $V$ & -1.25 & - & +1.93 & - & 1 & +3.28 & - & 1 \\
\hline & $R$ & -1.23 & - & +1.78 & - & 1 & +3.29 & - & 1 \\
\hline
\end{tabular}

observed ratio of the polarizations $\left(p_{B} / p_{V}=0.57\right)$ at the stellar elongations. Agreement with the observed ratio was attained for no test case. Moving the scattering concentration from the $L_{1}$ point closer to the hot star increased disagreement with the observed ratio. We conclude that less than half of the difference between the Oregon and FCO polarization amplitudes is due to the bandpass difference and that the rest is intrinsic. The concentration of scatterers must, therefore, have been variable in 


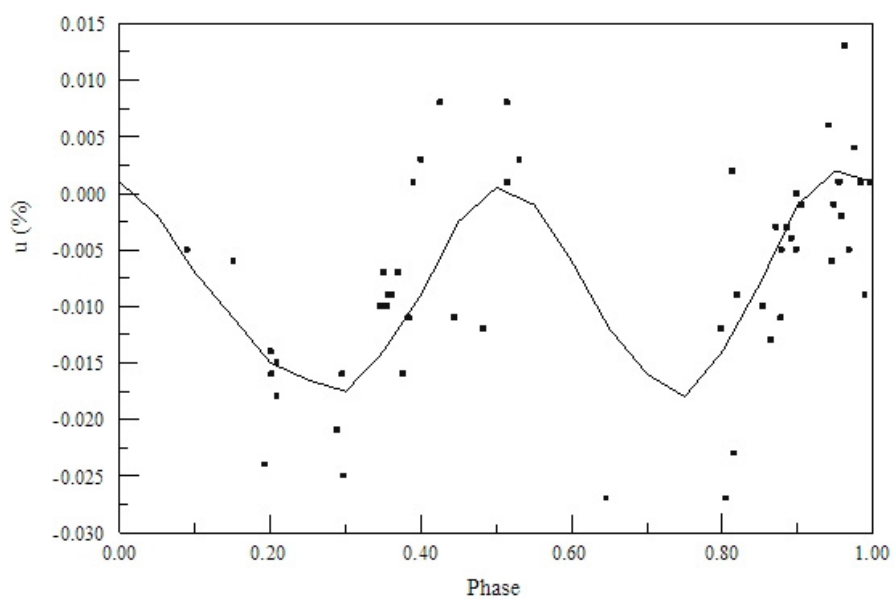

Fig. 5. The NLSP $u$ of $\beta$ Per as a function of orbital phase calculated from an ephemeris given by Kim (1980). The segmented curve connects the averaged data points (and not the smooth analytical fit) from Kemp et al. (1981) binned over phase intervals of $0.05 P$. Kemp et al. give an average error per measurement of $\pm 0.002 \%$. The average error per FCO measurement is $\pm 0.003 \%$.

density and/or geometry, indicating variability of the masstransfer process.

If the scatterers are not limited to electrons, the scattering will be a function of bandpass and a different conclusion might be reached if the cross section increases with decreasing wavelength. This fact may be important in the future since, as Richards (1992) showed, neutral atoms are not lacking in the mass-transferring stream.

The FCO $v$ measures are apparently the first ever attempted and one night showed a smooth variation before primary minimum with a peak-to-peak amplitude of $0.014 \%$. Since, however, there are no data after minimum, nothing can be inferred about this result. The NCSP showed no other modulation in or out of synchronism with the linear parameters, so it should be considered zero within its error as predicted by a comparison with Eq. (6).

\subsection{SZ Cam}

Our measures were taken on four nights in the 1991-1992 season and sample only four Keplerian phases. By analogy with other hot binaries, we expected that significant phase-locked variability would be evident but we saw none. Possibly the measures fall near the nodes of a double-cosine waveform. Or, perhaps the envelope is spherical and/or has a low density. The star was also observed twice by Coyne (1974) with a $B$ filter, leading to NLSP $q=+1.18 \% \pm 0.06 \%$ and NLSP $u=-5.96 \% \pm 0.10 \%$ (for standard deviations, multiply these errors by $\sqrt{2}$ ). Thus, there is no indication that this object is a long-term polarization variable. Equation (1) suggests that the standard deviation of a comparable standard star should be $\approx \pm 0.037 \%$, marginally supporting the possibility of variable, hence also intrinsic, polarization.

The visual companion to SZ Cam, ADS 2984B, was observed twice at the FCO, giving NLSP $q=+1.16 \% \pm 0.05 \%$ and NLSP $u=-6.05 \% \pm 0.01 \%$. All of Coyne's filtered measures show that the polarization is dominated by the interstellar component, and the FCO values for ADS 2984B may indicate an average interstellar polarization for NGC 1502, of which both stars are members. Alternatively, our measurements of "ADS 2984B" are very similar to Coyne's "SZ Cam" measurements. This fact, combined with the information that both stars are very similar in brightness, suggests a possible misidentification. The older, less precise pair of observations by Hall \& Mikesell (1950) does not resolve the confusion. All eight attempts to measure the NCSP failed.

\subsection{KS Per}

The observed standard deviations for the $B$ and $V$ means are consistent with a standard star of comparable brightness, so variability cannot be proven from the few measures collected in Table 1. We applied a spectroscopic ephemeris to our polarimetry, and it showed no phase-locked variability. The $p$ spectrum decreases with increasing wavelength, but not so fast as predicted by Rayleigh scattering. A significant fraction of the signal is likely to be interstellar because the distance is too great to have been measured by Hipparcos and $b=-1^{\circ}$.

\section{6. $\epsilon$ Aur}

The NLSP $q$ parameter in the $U$ bandpass differs significantly from the values in the other bandpasses. The difference may not be real because the instrumental correction in $U$ is significantly different from the others. The evidence from the $B, V$ and $R$ historical measures (Gehrels \& Sylvester 1965; Coyne \& Gehrels 1966) and the two tabulated $B$ values is that this atmosphericallyeclipsing binary is a polarization variable.

\section{7. $\alpha$ Aur (Capella)}

The noise levels for the 1st and 3rd measures are large because integration times were too brief. A spectroscopic ephemeris was used to compute phases. The star exhibited no phase-locked behavior. Since Capella is such a bright star, it has a significant observational history: $p=0.03 \%$ (Behr 1959), $p=0.00 \%$ (Tinbergen 1982), and $p=0.024 \%$ (Leroy 1993). The FCO mean would appear to be larger than these three examples, but its standard deviation is larger than those of Tinbergen (1982) and Leroy (1993), so there is no indication that it is a long-term polarization variable.

\subsection{AR Aur}

The individual measures are scattered from 1986 to 1991 and cover the cycle of this detached binary adequately without showing any phase-locked pattern. Equations (1) and (2) do not indicate variability. The fitting coefficients of Stewart (1991) were used to calculate unbiased maximim-likelihood estimates of the polarization. The bias-corrected result is only $p=0.008 \%$ and is not significant. Even though $b=-2^{\circ}$, the distance is only about $120 \mathrm{pc}$, so a result close to $p=0.00 \%$ is reasonable. The star might serve as a linear null standard. The mean circular component is also not significantly different from NCSP $v=0.00 \%$.

\section{9. $\eta$ Ori}

As isolated in the focal plane diaphragm, this multiple star embodies a pulsating object and a detached eclipsing binary (Lee et al. 1993). No phase-locked variability exists in the dataset and the mean agrees well with the historical observations by Behr (1959) and Hall \& Mikesell (1950). Equation (1) leads to exact agreement with the observed standard deviation. The small 
signal is likely interstellar, and this star could also serve as a low-polarization standard.

\subsection{LY Aur}

Pfeiffer (1975) first demonstrated the existence of a scattering envelope. The observed NLSPs in this work vary over the ranges $+0.37 \%<\Delta q_{V}<+0.52 \%$ and $-1.94 \%<\Delta u_{V}<-1.78 \%$, and there are numerous high-frequency transients superimposed on the approximate double-cosine waveform. Within the errors, these ranges are consistent with the old results of Hall \& Mikesell (1950), Hall (1958), and Hiltner (1951, 1954), so we claim no long-term variability. According to Eq. (2), this star should be a polarization variable. The observed polarization spectrum is dominated by the interstellar component with $q_{\text {is }, V}=+0.85 \%$ and $u_{\text {is }, V}=-1.47 \%$, leaving an intrinsic stellar polarization of the order of $p(V)=0.6 \%$. These interstellar values were obtained by choosing field stars within $\approx 5^{\circ}$ of LY Aur that were observed polarimetrically in surveys (Behr 1959; Hiltner 1951, 1951; Hall \& Mikesell 1950), plotting/fitting their $p$ and $\theta$ versus $m-M$, and plugging the $m-M$ of LY Aur into the fit. There is no significant rotation of the intrinsic electric vector with wavelength.

\subsection{1. $\delta$ Ori}

The means of Table 1 are calculated by giving half and full weight to the observations before and after JD 2444900 , respectively. The standard deviations are small, but by Eq. (1) this star is really variable over all time scales. During the years of FCO monitoring, there has been no enduring pattern of polarization change phase-locked to the eclipsing period. This result contradicts the early conclusion by Pfeiffer \& Koch (1990), although patterning of this kind does appear on a few nights. During the single season when Snow \& Hayes (1978) observed the binary, the NLSP $q$ was constant while the NLSP $u$ showed a first-harmonic variation of about $0.03 \%$ peak-to-peak. All of this behavior is likely due to the uncorrelated contributions from the assorted component stars (Harvin et al. 2002), which are unresolved by the polarimeter and hot enough to have individual scattering shells that are active and not oriented in a unique direction.

Multiple interpolation with Hipparcos distances and galactic coordinates for Orion stars gives well determined interstellar normalized Stokes parameters: $(-0.10 \%,+0.12 \%)$. These interstellar values were obtained by choosing field stars within $\approx 5^{\circ}$ of $\delta$ Ori that were observed polarimetrically in surveys (Behr 1959; Hiltner 1951, 1951; Hall \& Mikesell 1950), plotting/fitting their $p$ and $\theta$ versus $m-M$, and plugging the $m-M$ of $\delta$ Ori into the fit.

It is not unthinkable that the variable interstellar lines discovered by Price et al. (2001) could be accompanied by variable interstellar dust column densities. When the interstellar polarization is subtracted from the tabulated values, the net polarization is low compared to a nominal value of $p \approx 1 \%$ that might be expected for a hot, massive system. The interpretation of this anomaly is just as above, viz., the polarizations of the several unresolved stars add vectorially so that a net value can be small. When the interstellar components are removed, the change in the orientation of the electric vector (including the historical measures before the FCO program) is in the same retrograde sense and at about the same rate as the change of the polar angle of ADS 4134, of which $\delta$ Ori is the A component, that has been studied by visual astrometry since 1952 and by speckle interferometry since 1979.

\subsection{V395 Aur}

This 23-day ellipsoidal binary is clearly polarized, but the signal could be interstellar because of its low galactic latitude and distance of $250 \mathrm{pc}$. The UV and visible work by Dempsey et al. (1990) and Stickland (1991) show that there is much circumstellar gas in the system. Both authors agree, however, that the binary is not reddened, so any real polarization signal would have to be intrinsic. Possibly the one abundant set of Fe II circumstellar lines shows that there is gas which could be the source of scattering electrons. The observed standard deviations, however, are comparable to the predictions of Eqs. (1) and (2), and therefore our data cannot confirm NLSP variability. The observing runs spanned only 6 nights in 1993, so orbital coverage is poor, which may be a significant detail. On the basis of the observed $\mathrm{S} / \mathrm{N}$, the yellow CNPS might be real but the observed standard deviation is only as large as that expected by Eq. (6). Possibly all of this information could be reconciled if there exists a spherical absorbing and scattering shell but that option has no independent support.

\subsection{UX Mon}

The $U$ measure has a very large formal internal error and should be viewed skeptically until it is confirmed. The $V$ standard deviation is not large enough to indicate intrinsic polarization when compared to the standard deviation expected from Eq. (2), but there are so few observations that this is also not a convincing case for establishing polarization constancy, either.

\subsection{TX UMa}

Measures were obtained at Keplerian phases $0.13,0.27$ and 0.42 during early 1995 to test whether its polarization signal would be similar to Algol's. If there is a signal, it must be smaller than $0.03 \%$. We find no intrinsic polarization when the measured standard deviations are compared to Eqs. (2) and (1). At $b=+60^{\circ}$ and at a distance of only $200 \mathrm{pc}$, the interstellar polarization should be very small. The similarity between the evolutionary stages of Algol and TX UMa, however, strongly suggests that sufficiently numerous and accurate measures would show similar phase-locked modulation.

\subsection{5. $\delta$ Lib}

The standard deviation of the $B$ measures is comparable to Eq. (1), indicating that no intrinsic and variable signal has been detected. This binary is expected to show a pattern of variability similar to that of $\beta$ Per, but the $B$ measures are too few and have an unacceptable phase distribution to show if such a similarity exists.

\subsection{6. $\alpha \mathrm{CrB}$}

Previous independent determinations for this detached and unevolved binary have shown very small polarizations. It is true that the entries in the table are $\sim 0.00 \%$ within $3 \sigma$, but for such a bright star one would have expected smaller polarization values and standard deviations. The calculated standard deviation is significantly larger than the estimate of Eq. (1). The authors view 
this confounding result as the consequence of an observational or reduction error that cannot be explained.

A potential alternative to observing or calculating the standard deviation might be found in the extensive far-IR survey by Trilling et al. (2007). Therein, the observers found that a substantial fraction of A, Am, and F type unevolved or little-evolved spectroscopic binaries and light variables display the continuum flux excess characteristic of dust amalgamated into debris disks. Their range of Keplerian periods and semi-major axes encompass that of $\alpha \mathrm{CrB}$, so a non-uniform and time-variable dust distribution could cause the polarization variability that this binary appears to exhibit.

\subsection{V1143 Cyg}

This unevolved and detached binary shows the same unacceptable situation as $\alpha \mathrm{CrB}$. The two binaries were observed partly in the same season, which makes the expectation of an unknown observing or reduction error somewhat more credible. Other systems observed during the same seasons, however, do not exhibit this confusion. Because the stellar and orbital characteristics of this binary are so similar to those of $\alpha \mathrm{CrB}$, it is possible that the same polarization mechanism applies.

\subsection{V380 Cyg}

The FCO NLSP $u$ is somewhat noisier than the NLSP $q$. There are so few observations, however, that it is impossible to check for phase-locked variability. Neither NLSP agrees with Hall's (1958). Shakhovskoi's (1965) NLSP $q$ is similar to Hall's (1958), but his other NLSP is different; he actually considered his results to be null within the errors. V380 Cyg shows larger standard deviations compared to Eq. (1), which means that it may be variable.

\subsection{Cyg}

There appear to be no previously published measures for this star and nothing useful may be inferred from only two measures.

\subsection{Cyg}

Based on comparison with the standard-deviation equations, the polarization of this atmospherically eclipsing binary is intrinsic to the system and variable in all bandpasses. In the $B$ bandpass there appears a short-term pulse of about $p=0.2 \%$ peak-to-peak amplitude as shown in Fig. 6. The polarization spectrum is itself variable. Shakhovskoi's (1965) mean from a few observations does not agree with the mean of Table 1 , so this system is also a long-term variable.

\subsection{1. $H R 8281$}

Even though our data are few, the polarization measures form a phase-locked double-cosine curve with $\Delta q=0.3 \%$ and $\Delta u=$ $0.2 \%$ peak-to-peak amplitude. The binary has a well-developed stellar wind. A spectroscopic ephemeris was used to compute the phases since this $06+09$ system is not a light variable, but the observations are too few to delineate phase-locked modulation. The means in Table 1 are similar, within errors, to the few values in the old literature. Equation (1) is consistent with the star showing variable polarization.

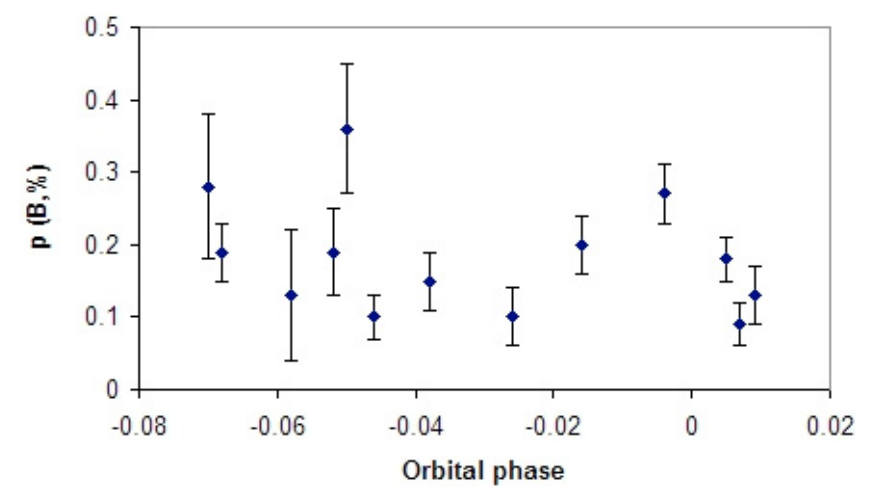

Fig. 6. The polarization of $32 \mathrm{Cyg}$ as a function of phase near primary eclipse, exhibiting the pulse.

\subsection{2. $A H$ Cep}

The first entry in the table for the $B$ data comes from the 1977 through 1980 seasons while the second entry comes from the 1982-1983 season. Each data set is noisy and neither shows a convincing phase-locked pattern. The $V$ and $R$ entries come from the 1977 season. Other workers have observed this star: $p=1.57 \% @ \theta=58.1^{\circ}$ in $U$ (Coyne 1974); $p=1.9 \% @$ $\theta=50^{\circ}$ in $B$ (Hall and Mikesell 1950); $p=1.62 \% @ \theta=57.7^{\circ}$ and $p=1.34 \% @ \theta=62.7^{\circ}$ in $V$ (Coyne 1974; and Shakhovskoi 1965 , respectively); and $p=0.91 \%$ @ $\theta=54.9^{\circ}$ in $R$ (Coyne 1974). In no bandpass does the polarization display constant long-term values. Equation (1) indicates variability for the earlier datasets and no variability for the 1982-1983 dataset. The interstellar polarization in this direction and at the distance of the binary $\left(p \approx 1.0 \% @ \theta \approx 85^{\circ}\right)$ is also very different from the observed values, so some systemic and variable source of polarization in this detached system is unknown at present. Most likely there is a variable wind from the stars.

\subsection{CW Cep}

One value of NLSP $q$ was ignored in calculating the mean for this parameter. Single-cosine waveforms with peak-to-peak amplitudes of $0.10 \%$ in NLSP $q$ and $0.07 \%$ in NLSP $u$ appear phase-locked to the Keplerian cycle. We found no double-cosine waveforms, but there are considerable phase gaps. Hiltner (1951) gives $p=0.7 \% @ \theta=37^{\circ}$ which, on the basis of his measures and errors for other stars, should be considered significantly different from the mean result of $p$ calculated from the NLSPs in Table 1. Equation (1) indicates that the standard deviation is greater than the standard deviation of a comparably bright standard star.

This binary is almost certainly a member of Cep OB3 (Clausen and Giménez 1991). The observed polarization is not aligned with the mean interstellar polarization of the association $\left(p \approx 2.4 \% \pm 1.2 \% @ \theta \approx 77^{\circ} \pm 22^{\circ}\right)$. These association values are not well determined because of the patchiness of the interstellar dust and the wind orientations of the member stars. Therefore, the mean association polarization may not provide an appropriate interstellar correction to the observed value for CW Cep. Pachoulakis et al. (1996) mapped discrete clouds above the photospheres of CW Cep using IUE SWP spectra. Pfeiffer et al. (1994) did the same for the comparable binary Y Cyg to supplement the earlier polarization measures of Koch \& Pfeiffer (1989). There appear to be sporadic episodes of variability in objects of this type. The standard deviation for the 
mean of the NCSP is not significantly different from that calculated with Eq. (6).

\subsection{AR Cas}

The number of observations is so few that cycle coverage is poor, but there may be a low-amplitude, phase-locked modulation. The mean FCO polarization is precise, and the NLSP $q$ value does not agree well with the mean of three observations $(q \approx 0.37 \%)$ by Hall \& Mikesell (1950). Equation (1) indicates that the system is a polarization variable.

\subsection{5. $\omega P s c$}

The NLSP $q$ was variable with time while NLSP $u$ was constant. According to Eq. (1), this star shows scatter significantly greater than a comparably bright standard star. The binarity of this object is uncertain, and the star appears to have no polarization history.

\subsection{V705 Cas (Nova Cas 1993)}

This object was observed on the third day after maximum light. Even though only one measure was made in each bandpass, linear interpolation among the photometry of Munari et al. (1993) leads to estimated standard deviations of $\pm 0.02 \%$ and $\pm 0.03 \%$ using Eqs. (1) and (2). The results of Hauschildt et al. (1994) and Evans et al. (1996) assure that the polarization measures were taken before dust formation. From the $B$ interstellar value given by Evans et al. (2002), we thus find intrinsic polarization very early in the outburst and definitely in the pre-dust interval. These conclusions are consistent with the observed polarization spectrum, which is not interstellar.

The tabulated values are also very different from El'kin's (1995), taken on day +26 after maximum light. The polarization level and spectral changes are likely due to emission features which vary with time and bandpass. Presumably, the intrinsic signal arises from an asymmetrical and/or inhomogeneous emitting shell since we know that the shell contained no dust at the early time reported here.

There is no independent check of the very large $v$ values with estimated errors of $\pm 0.02 \%$. Through the history of the FCO polarimetry, no other object ever showed so large a value of $v$ except for SX Cas (Elias 1990). Despite the integrity of the instrumental calibration, the large values for this star and SX Cas should be viewed skeptically.

\subsection{Variability summary}

Two objects show constant polarization, AR Aur $(\approx$ null $)$ and $\eta$ Ori. At least thirteen other targets (LY Aur, $\epsilon$ Aur, AR Cas, V705 Cas, AH Cep, CW Cep, V380 Cyg, 32 Cyg, $\delta$ Ori, $\beta$ Per, $\omega$ Psc, and HR 8281) are confirmed to be variable over orbital and longer timescales.

Information about the remaining binaries is too meager or confusing for a decision about variability, but analogs among other close binaries of comparable classes suggest that most of these will eventually be confirmed variable as well. For the most part, when the three possible tests for variability could be applied to a binary, they gave consistent results. Conspicuous failures of the procedure occurred for $\alpha \mathrm{CrB}$ and V1143 Cyg.

None of the results herein refer to the numerous FCO measures of over-contact binaries which remain unpublished. There are also substantial unpublished data sets for LZ Cep, $\beta$ Lyr, and V367 Cyg.

\section{Hot non-radially pulsating stars}

Between 1987 and 1990, the first author conducted a dedicated program monitoring a selection of $\beta$ Cepheid stars. According to Clarke (1986), these non-radial pulsators may exhibit sinusoidal polarization behavior as a function of pulsational phase. A summary of the results for these stars appears in Table 2, which is structured nearly in the same manner as Table 1 . The only difference is that all the measures except for one were obtained in the $B$ filter, so there is no filter column. The reduced standard deviations must be multiplied by the square root of the number of measurements for comparison with the standard deviations of polarized standard stars.

Because they are relatively bright and hot, most of these stars have some polarization histories from the earliest surveys. These provide at least a minimum context for the measures given here. Comments about the internal and external consistencies of our data appear in the following subsections.

\section{1. $88 \gamma$ Peg (Algenib)}

The entry for NLSP $u$ in the table disguises some detail. The mean of seventeen measures in the 1988 season is $-0.027 \% \pm$ $0.002 \%$ while the similar mean of eight measures in 1990 is $+0.007 \% \pm 0.006 \%$. The 1988 data were obtained on multiple nights, while the 1990 data were obtained on a single night. No star in Tables 1 or 2 shows such a discontinuity in time although the time span for this star is by far the greatest of all targets in Table 2. Even though these changes are small, they far exceed the errors.

The only other non-null measure of which we are aware is by Behr (1959): $p=0.069 \% @ \theta=124^{\circ}$, which, within errors, is not dissimilar to the mean from 1988: $p=0.035 \% @ \theta=116^{\circ}$. Hall's (1958) two measures were unable to find a signal above the measurement errors. Whatever the cause of the discrepancy, it is presumed to be an intrinsic characteristic of this object.

We have also found tentative evidence for phase-locked behavior. In Figs. 7 and 8, we plot NLSP $q$ and NLSP $u$ for the two seasons and barely see sinusoidal behavior. The variability is more pronounced in NLSP $u$. The NLSP $u$ fits for both seasons are

$$
\begin{aligned}
u(\phi)= & -0.054( \pm 0.003)+0.003( \pm 0.005) \cos \phi \\
+ & 0.001( \pm 0.004) \sin \phi \quad(\text { season } 1)
\end{aligned}
$$

and

$$
\begin{aligned}
u(\phi)= & -0.013( \pm 0.002)+0.003( \pm 0.002) \cos \phi \\
+ & 0.001( \pm 0.003) \sin \phi \quad(\text { season } 2)
\end{aligned}
$$

where $\phi$ is the orbital phase. Unfortunately, the amplitudes are small with respect to their formal errors. This object is worth additional study using a sensitive instrument with a well defined (small and constant) instrumental polarization.

\section{2. $53 \cup W$ Ari}

The standard deviation predicted by Eq. (1) for $B=+6.00$ is consistent with the calculated standard deviation. Hall (1958) measured $p=0.51 \% @ \theta=135^{\circ}$, in reasonable agreement with 
Table 2. The weighted means of the NLSPs and NCSPs, together with the reduced standard deviations and number of observations. All measurements are in $B$ except for a single $V$ measurement of V600 Her (indicated). The reduced standard deviations must be multipled by the square root of the number of measurements to compare them with the standard-deviation equation.

\begin{tabular}{lcccccccc}
\hline \hline Name & $\begin{array}{c}q \\
(\%)\end{array}$ & $\begin{array}{c}\sigma_{q} \\
(\%)\end{array}$ & $\begin{array}{c}u \\
(\%)\end{array}$ & $\begin{array}{c}\sigma_{u} \\
(\%)\end{array}$ & $N_{\text {lin }}$ & $\begin{array}{c}v \\
(\%)\end{array}$ & $\begin{array}{c}\sigma_{v} \\
(\%)\end{array}$ & $N_{\text {circ }}$ \\
\hline$\gamma$ Peg / HD 886 (season 1) & -0.030 & 0.008 & -0.054 & 0.006 & 17 & -0.006 & 0.009 & 17 \\
$\gamma$ Peg / HD 886 (season 2) & -0.024 & 0.003 & -0.016 & 0.003 & 8 & -0.002 & 0.004 & 8 \\
UW Ari / HD 19374 & -0.010 & 0.006 & -0.540 & 0.010 & 21 & -0.002 & 0.008 & 15 \\
RS Sex / HD 89688 & -0.150 & 0.010 & +0.230 & 0.010 & 9 & +0.10 & 0.05 & 2 \\
$\alpha$ Vir / HD 116658 & -0.0134 & 0.0009 & -0.026 & 0.002 & 11 & -0.010 & 0.002 & 5 \\
V600 Her / HD 149881 & -0.433 & 0.008 & +0.123 & 0.009 & 27 & -0.02 & 0.02 & 27 \\
V600 Her / HD 149881 (green) & -0.310 & 0.010 & +0.050 & 0.010 & 1 & +0.06 & 0.09 & 1 \\
ES Vul / HD 180968 & +0.163 & 0.006 & +0.441 & 0.006 & 9 & -0.02 & 0.01 & 8 \\
BW Vul / HD 199140 & +0.244 & 0.008 & +0.309 & 0.009 & 10 & -0.026 & 0.008 & 9 \\
12 DD Lac / HD 214993 & -0.406 & 0.005 & +0.124 & 0.005 & 13 & -0.017 & 0.009 & 9 \\
16 EN Lac / HD 216916 & -0.319 & 0.007 & +0.002 & 0.007 & 18 & -0.006 & 0.006 & 10 \\
LN And / HD 217811 & -1.07 & 0.06 & +0.55 & 0.01 & 2 & -0.05 & 0.02 & 2 \\
LQ And / HD 224559 & +0.102 & 0.009 & +0.483 & 0.009 & 19 & -0.000 & 0.010 & 19 \\
\hline
\end{tabular}

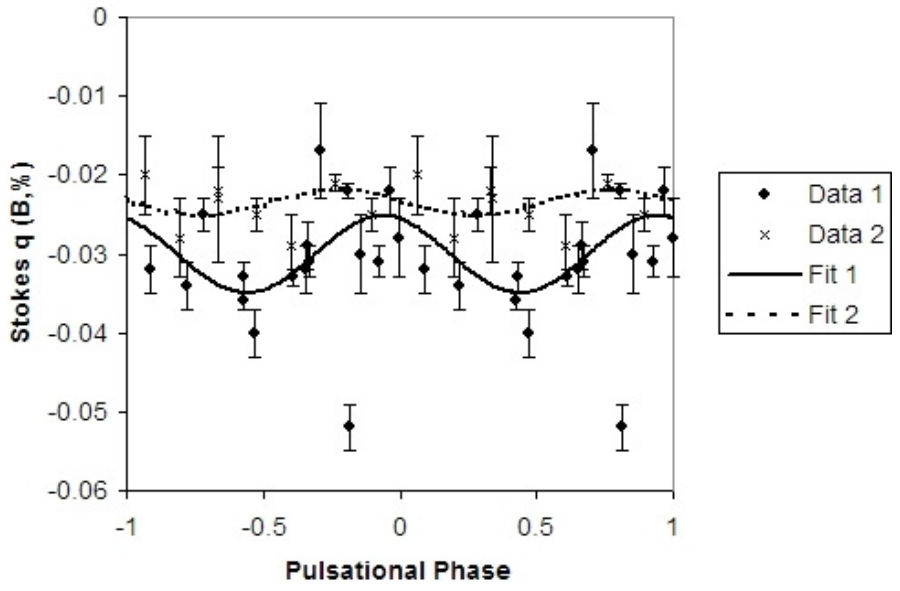

Fig. 7. The NLSP $q$ of $88 \gamma$ Peg as a function of pulsational phase for two separate seasons.

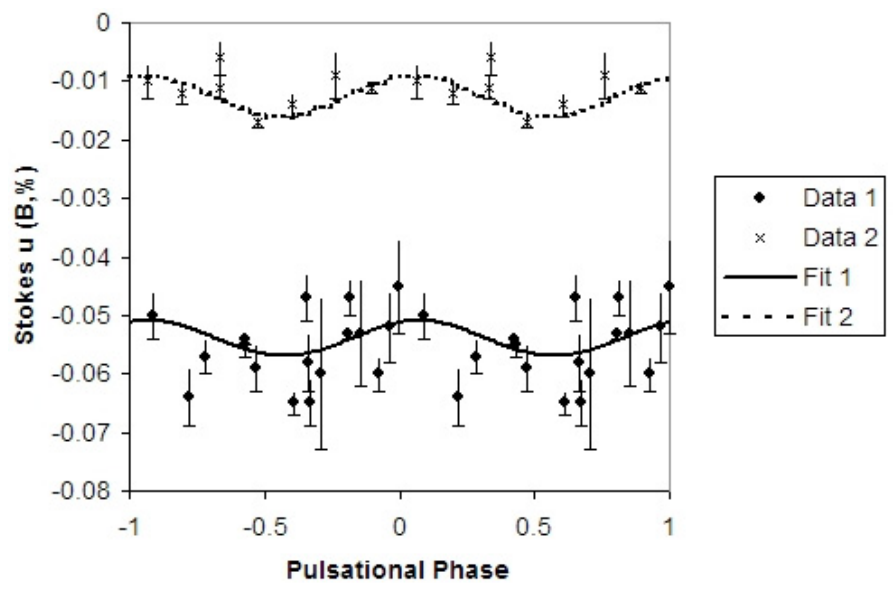

Fig. 8. The NLSP $u$ of $88 \gamma$ Peg as a function of pulsational phase for two separate seasons.

our result. The SIMBAD database cites this star as a spectroscopic binary so this system could have appeared in the previous section.

\subsection{RS Sex}

According to Eq. (1), the observed standard deviation is exactly the value expected for a non-variable star. The polarization is, therefore, constant and non-zero. Four observations by Hall (1958) failed to obtain a measure significantly above their observational errors, a discrepancy which cannot be explained.

\section{4. $67 \alpha \operatorname{Vir}($ Spica)}

The standard deviation predicted by Eq. (1) indicates that this star is not a polarization variable. Hall (1958) could not measure the polarization of Spica within his errors, but Behr did succeed in obtaining $p=0.01 \%$ @ $\theta=147^{\circ}$, and Pfeiffer \& Koch (1977) recorded $p=0.03 \%$ in a program much earlier than the results presented here. In view of the very small signal, this early result may be considered acceptable agreement with the mean in Table 2. Spica could have been included among the close binaries of Table 1 and, in view of its hot temperature, expected to show a scattering envelope. Failure to detect a variable signal could be the result of poor phase sampling with the Keplerian period so close to four days.

\section{5. $\mathrm{V} 600 \mathrm{Her}$}

This $\beta$ Cepheid star is in a short-period binary with another early-type star (Hill et al. 1976). Although the measured standard deviations are larger than predicted by Eq. (1), they are not sufficiently large to classify this star as a polarization variable. Again, Hall (1958) published a null result, but Mathewson and Ford (1970) record $p=0.46 \% @ \theta=85.9^{\circ}$. Their observation is in excellent agreement with the mean of Table 2. There is no indication that the $B$ and $V$ measures are inconsistent with interstellar polarization.

\subsection{ES Vul}

The measure by Hiltner (1951) is $p=0.74 \% @ \theta=27^{\circ}$ and is $0.27 \%$ larger and rotated by $-8^{\circ}$ compared to the mean given in the table. The standard deviation tabulated in Table 2 is smaller than predicted by Eq. (1), so this object has to be considered a constant-polarization star over short time scales. This star has been listed as a possible Be star by Percy et al. (2002), which 
could explain the long-term changes. There is no evidence for variability at the pulsational period.

\subsection{BW Vul}

The standard deviation estimated by Eq. (1) is in essential agreement with the standard deviation in Table 2, so there is no evidence of variability. Hall (1958) gives $p=0.28 \% @ \theta=$ $66^{\circ}$ from two observations. While the angle is in good agreement with the value tabulated here, the old polarization value is smaller than ours by about $0.11 \%$. This discrepancy, however, is comparable to Hall's measurement error.

\subsection{DD Lac}

This $\beta$ Cepheid star is in a short-period binary. Hall (1958) was unable to find a non-zero result. We found no evidence for variability versus pulsational or orbital phase using Eq. (1).

\subsection{EN Lac}

The standard deviations in the table are almost identical to those predicted by Eq. (1), so the star is not a polarization variable. Four observations by Hall (1958) did not yield a significant nonzero mean, a surprising result in view of his observational errors and the mean magnitude.

\subsection{LN And}

A value of $p=0.97 \% @ \theta=82^{\circ}$ is given by Hall (1958), and may be considered in marginal agreement with the result of $p=$ $1.20 \% @ \theta=76^{\circ}$ listed above.

\subsection{1. $L Q$ And}

Hall (1958) observed $p=0.64 \% @ \theta=20^{\circ}$, whereas we find $p=0.49 \% @ \theta=39^{\circ}$. In view of his larger errors, on the order of $0.1 \%$, the comparison shows acceptable agreement.

\subsection{Variability summary}

These results may be summarized as follows. Of the eleven program stars, two of them ( $\gamma$ Peg, ES Vul) give some indication of short- or long- term variability of the NLSP values. In particular, ES Vul may be a Be star. According to Eq. (6), none of these targets exhibits circular polarization.

The usual signatures of $\beta$ Cepheid pulsating stars are smallscale light, radial velocity, and line profile variabilities, but not extravagant indicators such as strong spectral emissions. Possibly using only the fundamental pulsation period, as was done here, failed to uncover a signal phase-locked to other excited pulsations or to beat periods among them. Some of these targets are members of close binaries, but our polarization measures do not show dependence on orbital phase. Apart from ES Vul, we do not need to invoke circumstellar shells.

\section{Linear polarization versus extinction}

Since both interstellar polarization and extinction are produced by interstellar dust grains, logically we would expect them to be correlated. In Fig. 9, we plot the $B$ polarization versus reddening for all of the standard and close-binary stars discussed in this paper. The former have already been published by

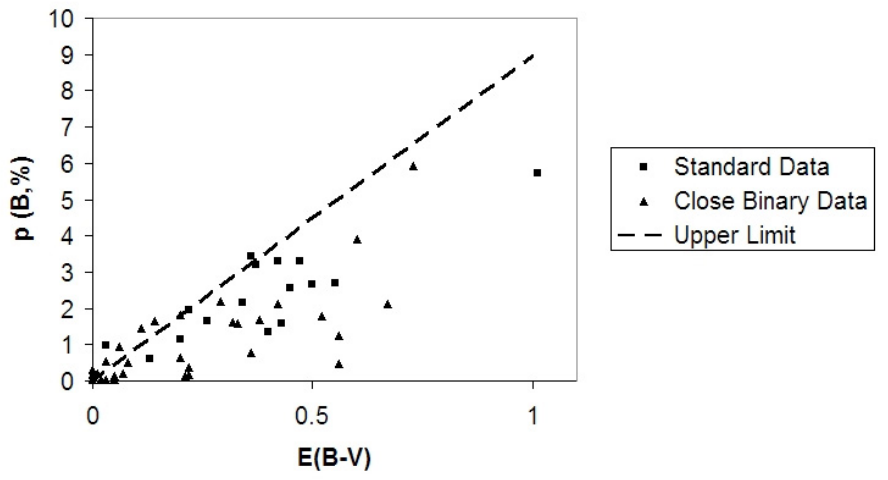

Fig. 9. The dependence of $B$ polarization on interstellar reddening for standards and close binaries. V395 Aur was not included because it is unclear what intrinsic color index should be assigned to it. More than 25 null polarization standards given by Koch \& Clarke (2005) would essentially occupy the origin but are not plotted.

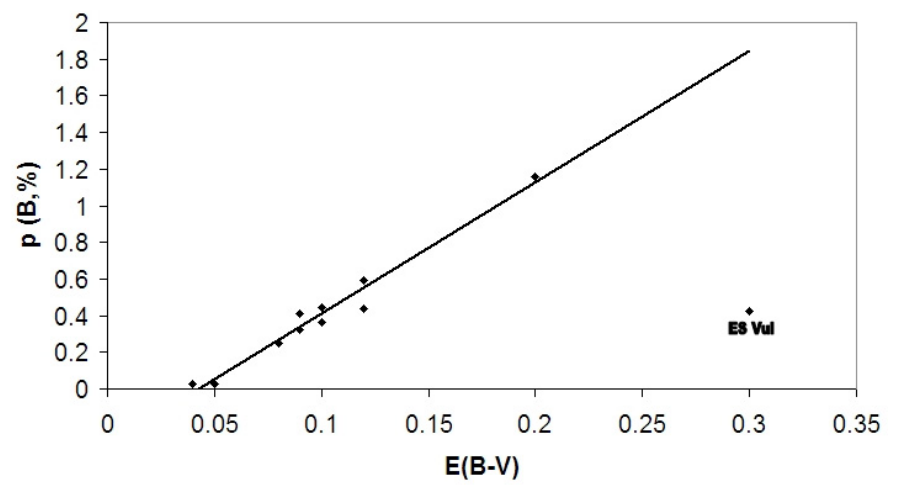

Fig. 10. The dependence of $B$ polarization on interstellar reddening for $\beta$ Cepheid stars. ES Vul was not included in the fit because it could be a Be star.

Koch (1990, 2006) and Koch \& Clarke (2005). Serkowski et al. (1975) create a similar plot and find an empirical upper limit

$p_{\text {max }} \lesssim 9 E(B-V)$,

where $p_{\max }$ is the maximum value in the polarization spectrum. We display this line in our plot, but note that our abscissa is the $B$ polarization which can be slightly different from $p_{\max }$. The distribution is characteristic of the intrinsic polarization of the close-binary stars, the clumpiness of the dust in the interstellar medium, as well as the different orientations of the galactic magnetic field with respect to the lines of sight.

In Fig. 10, we present the same type of plot for only the $\beta$ Cepheid stars. We find that all but one of the stars (ES Vul, a possible Be star) fall tightly on the line

$p(B)=-0.26( \pm 0.14)+6.37( \pm 1.33) E(B-V)$,

whose slope is considerably less than nine. We find this result very surprising. Granted, these relatively nearby stars form a very homogeneous set with small intrinsic polarizations, but we would expect to find the same distribution seen in Fig. 9 since these stars are located on very different lines of sight (ranges in galactic coordinates: $\Delta l=285^{\circ}$ and $\Delta b=93^{\circ}$ ). It is possible that this small group of stars has conspired to give a good fit to a straight line, but only observations of more $\beta$ Cepheid stars will confirm this hypothesis. If more observations preserve the linear behavior, $\beta$ Cepheid stars could act as excellent tracers of the interstellar medium. 


\section{Conclusions}

We have summarized optical polarimetry of close binaries and $\beta$ Cepheids from the Flower and Cook Observatory, and make them available via an on-line table. Using the standard stars, we fitted the polarization standard deviations as functions of apparent magnitude and used the results to ascertain the variability or non-variability of the program stars. Also, we examined the polarization of the program stars versus orbital and pulsational phases to search for variability.

Using our tests for variability, we: 1) confirmed the scattering cloud at $L_{1}$ for $\beta$ Per; 2) confirmed scattering envelopes around LY Aur and HR 8281; 3) discovered intrinsic polarization as early as day +3 after explosion for Nova Cas 1993; 4) discovered NLSP variability near primary eclipse of 32 Cyg; and 5) discovered long-term and possible phase-locked variability for $\gamma$ Peg. Also, we tentatively found a tight linear relationship between NLSP and $E(B-V)$ for $\beta$ Cepheid stars.

Acknowledgements. J. D. Dorren contributed a number of the early measures of AH Cep, and J. T. Feo measured the $V$ and $R$ observations for this target. M. al-Bussaidi helped to observe CW Cep. The authors would also like to thank the anonymous referee and the editor S. N. Shore for their comments and suggestions.

\section{References}

Bastien, P., Vernet, E., Drissen, L., et al. 2007, PASP Conf. Ser., 364, 529 Behr, A. 1959, VeGoe, 126, 196

Clarke, D. 1986, A\&A, 161, 412

Clausen, J. V., \& Giménez, A. 1991, A\&A, 241, 98

Coyne G. V. 1974, AJ, 79, 565

Coyne, G. V., \& Gehrels, T. 1966, AJ, 71, 355

Dempsey, R. C., Bopp, B. W., Parsons, S. B., \& Fekel, F. C. 1990, PASP, 102, 312

Elias, N. 1990, Ph.D. Thesis, University of Pennsylvania

El'kin, V. G. 1995, AstrL, 21, 670

Evans, A., Geballe, T. R., Rawlings, J. M. C., \& Scott, A. D. 1996, MNRAS, 282, 1049
Evans, A., Yudin, R. V., Naylor, T., Ringwald, F. A., \& Koch Miramond, L. 2002, A\&A, 384, 504

Gehrels, T., \& Sylvester, A. B. 1965, AJ, 70, 579

Hall, J. S. 1958, PubUSNO, 17(I)

Hall, J. S., \& Mikesell, A. H. 1950, PubUSNO, 17(I), 21

Harvin, J. A., Gies, D. R., Bagnuolo, W. G., Penny, L. R., \& Thaller, M. L. 2002, ApJ, 565, 1216

Hauschildt, P. H., Starrfield, S., Shore, S. N., et al. 1994, AJ, 108, 1008

Hill, G., Odgers, G. J., \& Drolet, B. 1976, A\&A, 51, 1

Hiltner, W. A. 1951, ApJ, 114, 241

Hiltner, W. A. 1954, ApJ, 120, 454

Kemp, J. C., Barbour, M. S., McBirney, R. E., \& Rudy, R. J. 1981, ApJ, 243, 557

Kim, H.-I. 1980, ApJ, 242, 1061

Koch, R. H. 1990, IBCDS, 38, 175

Koch, R. H. 2006, Obs, 126, 182

Koch, R. H., \& Clarke, D. 2005, Obs, 125, 355

Koch, R. H., \& Pfeiffer, R. J. 1989, PASP, 101, 279

Kreiner, J. M., Kim, C.-H., \& Nha, I.-S. 2001, An Atlas of O-C Diagrams of Eclipsing Binary Stars (Krakow: Wydawn. Nauk. Akad. Pedagogicznej)

Lee, W. B., Sung, E. C., Koch, R. H., et al. 1993, PASP Conf. Ser., 38, 239

Leroy, J. L. 1993, A\&AS, 101, 551

Mathewson, D. S., \& Ford, V. L. 1970, MemRAS, 74, 139

Munari, V., Tomov, T. V., Antonov, A., et al. E. 1993, IBVS, 4005, 1

Pachoulakis, I., Pfeiffer, R. J., Koch, R. H., \& Stickland, D. J. 1996, Obs, 115, 89

Percy, J. R., Hosick, J., Kincaide, H., \& Pang, C. 2002, PASP, 114, 551

Pfeiffer, R. J. 1975, Ph.D. Thesis, University of Pennsylvania

Pfeiffer, R. J., \& Koch, R. H. 1977, PASP, 89, 147

Pfeiffer, R. J., \& Koch, R. H. 1990, BAAS, 22, 1295

Pfeiffer, R. J., Pachoulakis, I., Koch, R. H., \& Stickland, D. J. 1994, Observatory, 114, 297

Price, R. J., Crawford, I. A., Barlow, M. J., \& Howarth, I. D. 2001, MNRAS, 328,555

Richards, M. T. 1992, ApJ, 387, 329

Scargle, J. D. 1982, ApJ, 263, 835

Serkowski, K., Mathewson, D. M., \& Ford, V. L. 1975, ApJ, 196, 261

Shakhovskoi, N. M. 1965, SovAstr, 8, 833

Snow, T. P., \& Hayes, D. P. 1978, ApJ, 226, 897

Stewart, B. G. 1991, A\&A, 246, 280

Stickland, D. J. 1991, Obs, 111, 225

Tinbergen, J. 1982, A\&A, 105, 53

Trilling, D. E., Stansberry, J. A., Stapelfeldt, K. R., et al. 2007, ApJ, 658, 1289 